University of New Orleans

ScholarWorks@UNO

$11-19-2008$

\title{
Global Existence of Some Infinite Energy Solutions for a Perfect Incompressible Fluid
}

\author{
Ralph A. Saxton \\ University of New Orleans, rsaxton@uno.edu \\ Feride Tiğlay
}

Follow this and additional works at: https://scholarworks.uno.edu/math_facpubs

Part of the Applied Mathematics Commons

\section{Recommended Citation}

Global Existence of Some Infinite Energy Solutions for a Perfect Incompressible Fluid Ralph Saxton and Feride Tiglay, SIAM J. Math. Anal. 40, 1499 (2008), DOI:10.1137/080713768

This Article is brought to you for free and open access by the Department of Mathematics at ScholarWorks@UNO. It has been accepted for inclusion in Mathematics Faculty Publications by an authorized administrator of ScholarWorks@UNO.For more information, please contact scholarworks@uno.edu. 


\title{
GLOBAL EXISTENCE OF SOME INFINITE ENERGY SOLUTIONS FOR A PERFECT INCOMPRESSIBLE FLUID*
}

\author{
RALPH SAXTON ${ }^{\dagger}$ AND FERIDE TIĞLAY ${ }^{\dagger}$
}

\begin{abstract}
This paper provides results on local and global existence for a class of solutions to the Euler equations for an incompressible, inviscid fluid. By considering a class of solutions which exhibits a characteristic growth at infinity we obtain an initial value problem for a nonlocal equation. We establish local well-posedness in all dimensions and persistence in time of these solutions for three and higher dimensions. We also examine a weaker class of global solutions.
\end{abstract}

Key words. incompressible fluid, three dimensions, stagnation point, global existence, Cauchy problem for periodic initial data, infinite energy

AMS subject classifications. 35Q35, 76B03

DOI. $10.1137 / 080713768$

1. Introduction. A fundamental question in the study of fluids concerns the possibility of finite time blow up of solutions to the Euler equations for a perfect, incompressible fluid. It is well known that blow up cannot take place in the twodimensional case for solutions defined over a bounded domain subject to Dirichlet boundary conditions (see, for example, the work of Wolibner [17] and Ebin [7]), since smooth data in this case lead to solutions remaining smooth for all time. However, the question remains open in higher dimensions.

A separate class of solutions consists of those having "stagnation-point" form, which attracted early attention by Weyl [16] and Lin [13], and provides a set of equations which depend on only a single spatial variable and time. The resulting equations, once solved, provide exact solutions to the full Euler equations. However, the associated growth of the full solutions in certain directions means that the flows possess, at best, only locally finite kinetic energy. Nevertheless, one may discuss such questions as finite time blow up for this class, and it has been shown by Stuart in [15] that this can take place when the reduced equations are defined over the real line and those solutions decay at infinity. In this case the corresponding spatial domain for the full equations is $\mathbb{R}^{n}$ for $n=2$ and $n=3$, with the full set of solutions growing linearly in the other direction(s).

The evolution of two-dimensional solutions, which can blow up on the unbounded domain $\mathbb{R}^{2}$, therefore differs significantly from the class of globally defined solutions which exists for bounded subdomains of $\mathbb{R}^{2}$. So the consequences of higherdimensional stagnation-point solutions blowing up might be thought to result simply from their behavior at infinity rather than bearing on the question of singularity formation. This view is in some sense strengthened by the results of Childress et al. in [3] (see also a related result by Cox in [5]), where solutions defined over a twodimensional, infinite strip were examined. Since blow up was still found over this, smaller, semi-infinite domain, stagnation-point solutions defined over such domains

\footnotetext{
*Received by the editors January 18, 2008; accepted for publication (in revised form) May 27, 2008; published electronically November 19, 2008.

http://www.siam.org/journals/sima/40-4/71376.html

${ }^{\dagger}$ Department of Mathematics, University of New Orleans, New Orleans, LA 70148 (rsaxton@ math.uno.edu, ftigley@uno.edu). The second author carried out part of this work at the Department of Mathematics at the University of Notre Dame.
} 
would give the appearance of behaving, generally, much as those defined on the full space.

A similar approach is implemented by Constantin in [4] to reduce the threedimensional Euler equations, periodic in two directions, to a nonlocal Riccati equation and prove the blow up in finite time by solving these equations on characteristics.

In this paper we consider a stagnation-point class of solutions defined over $\mathbb{R}^{n}$ which is spatially periodic in one coordinate direction. In two dimensions, the equations reduce to those of [3] and, although we examine slightly different boundary conditions, the same blow up results essentially apply. In three and higher dimensions, however, we find a "regularizing" effect not present in solutions which decay to zero at infinity in the same coordinate direction, and this leads to the existence for all time of all such solutions, stemming from sufficiently smooth initial data.

Section 2 sets out the fundamental field equations, which in a basic sense date back to [13], [16]. Section 3 is devoted to local well-posedness (existence, uniqueness, and continuous dependence on initial data) of classical solutions to the initial value problem for the pseudodifferential equation derived in section 2. We establish this result by rewriting the problem on the topological group $\mathcal{D}$ of $C^{1}$ class diffeomorphisms as an initial value problem for an ordinary differential equation. Section 4 provides a priori estimates for more regular classes of solutions, leading to global existence of such solutions in three and higher dimensions.

In section 5, we reconsider a class of piecewise affine solutions previously mentioned in [3]. These solutions are less regular than those arising in our existence results. It is found that they exist globally, independently of the underlying dimension.

2. The $n$-dimensional equation. Consider the $n$-dimensional Euler equations for an ideal, inviscid and incompressible fluid

$$
\begin{aligned}
\partial_{t} \mathbf{u}+\mathbf{u} . \nabla \mathbf{u}+\nabla p & =0, \\
\nabla \cdot \mathbf{u} & =0,
\end{aligned}
$$

where $\mathbf{x}=\left(x_{1}, \ldots x_{n}\right) \equiv\left(x_{1}, \mathbf{x}^{\prime}\right)$. Denoting $x_{1}$ by $x, \mathbf{u}\left(x, \mathbf{x}^{\prime}, t\right)$ represents the spatial velocity field of the fluid and $p\left(x, \mathbf{x}^{\prime}, t\right)$ its pressure. We impose the ansatz

$$
\mathbf{u}\left(x, \mathbf{x}^{\prime}, t\right)=\left(u(x, t),-\partial_{x} u(x, t) \mathbf{v}\left(\mathbf{x}^{\prime}, t\right)\right),
$$

where the $(n-1)$-dimensional vector field, $\mathbf{v}$, will be chosen below. As a consequence of (2.2), (2.1a) may be written as

$$
\partial_{t} u+u \partial_{x} u+\partial_{x} p=0
$$

together with

$$
\partial_{t} \partial_{x} u \mathbf{v}+\partial_{x} u \partial_{t} \mathbf{v}+u \partial_{x}^{2} u \mathbf{v}-\left(\partial_{x} u\right)^{2} \mathbf{v} \cdot \nabla^{\prime} \mathbf{v}-\nabla^{\prime} p=\mathbf{0},
$$

where the primed operators refer to the variable $\mathbf{x}^{\prime}$. Using (2.2) and (2.3), one sees that $\nabla^{\prime} \partial_{x} p=0$. Hence, differentiating (2.4) in $x$ eliminates the pressure term to give

$$
\partial_{x}\left(\partial_{t} \partial_{x} u+u \partial_{x}^{2} u\right) \mathbf{v}+\partial_{x}^{2} u \partial_{t} \mathbf{v}-\partial_{x}\left(\left(\partial_{x} u\right)^{2}\right) \mathbf{v} \cdot \nabla^{\prime} \mathbf{v}=\mathbf{0} .
$$

Applying the $\nabla^{\prime}$. operator to $(2.5)$ and using $(2.1 \mathrm{~b})$ with $(2.2)$ to find that $\nabla^{\prime} \cdot \mathbf{v}=1$ shows

$$
\partial_{x}\left(\partial_{t} \partial_{x} u+u \partial_{x}^{2} u\right)-\partial_{x}\left(\left(\partial_{x} u\right)^{2}\right) \nabla^{\prime} \mathbf{v}: \nabla^{\prime} \mathbf{v}=0,
$$


where $\nabla^{\prime} \mathbf{v}: \nabla^{\prime} \mathbf{v}=\operatorname{tr}\left(\nabla^{\prime} \mathbf{v}\right)^{2}=\partial_{j} v_{k} \partial_{k} v_{j}$ (summing over $j, k$ from 2 to $n$ ). For compatibility, we must choose $\mathbf{v}$ such that $\nabla^{\prime} \mathbf{v}: \nabla^{\prime} \mathbf{v}$ is independent of $\mathbf{x}^{\prime}$. This can be done, for instance, by choosing $\mathbf{v}=\frac{1}{n-1} \mathbf{x}^{\prime}$, in which case $\nabla^{\prime} \mathbf{v}: \nabla^{\prime} \mathbf{v}=\frac{1}{n-1}$ and (2.4) takes the form

$$
\left(\partial_{t} \partial_{x} u+u \partial_{x}^{2} u-\frac{1}{n-1}\left(\partial_{x} u\right)^{2}\right) \mathbf{v}-\nabla^{\prime} p=\mathbf{0} .
$$

We note that this particular choice of $\mathbf{v}$ corresponds to the stagnation-point form solution referred to in the introduction. As a result, the solution becomes unbounded in the $\mathbf{x}^{\prime}$ direction, and hence has infinite energy when considered over the entire $n$ dimensional domain. We next examine the periodic, initial-boundary value problem, with boundary conditions for $x \in \mathbb{T} \simeq \mathbb{R} / \mathbb{Z}, t \geq 0$, given by

$$
\mathbf{u}\left(0, \mathbf{x}^{\prime}, t\right)=\mathbf{u}\left(1, \mathbf{x}^{\prime}, t\right)
$$

and

$$
p\left(0, \mathbf{x}^{\prime}, t\right)=p\left(1, \mathbf{x}^{\prime}, t\right)
$$

Since (2.6) now becomes

$$
\left.\partial_{x}\left(\partial_{t} \partial_{x} u+u \partial_{x}^{2} u\right)-\frac{1}{n-1} \partial_{x}\left(\left(\partial_{x} u\right)^{2}\right)\right)=0,
$$

which we remark happens to be the $x$-derivative of a Calogero-class equation (see [2])

$$
\partial_{x} \partial_{t} u+u \partial_{x}^{2} u-\Phi\left(\partial_{x} u\right)=0
$$

we obtain the equation

$$
\partial_{t} \partial_{x} u+u \partial_{x}^{2} u-\frac{1}{n-1}\left(\partial_{x} u\right)^{2}=f
$$

with $f$ purely a function of time. This implies, by (2.7), that

$$
\nabla^{\prime} p=\frac{f}{n-1} \mathbf{x}^{\prime}
$$

while by $(2.3),-\partial_{x}^{2} p=\frac{n}{n-1}\left(\partial_{x} u\right)^{2}+f$ and so $\Delta p=-\frac{n}{n-1}\left(\partial_{x} u\right)^{2}$. Finally, for sufficiently smooth functions $u(x, t)$, using $(2.8)$ and integrating (2.11) we have

$$
f=-\frac{n}{n-1} \int_{\mathbb{T}}\left(\partial_{x} u\right)^{2} d x
$$

while (2.3), (2.8), and (2.9) imply that

$$
\frac{d}{d t} \int_{\mathbb{T}} u d x=0
$$

Let us introduce the operator $\partial_{x}^{-1}$ defined by

$$
\partial_{x}^{-1} \phi(x, t)=\int_{x_{0}}^{x} \phi(y, t) d y-\int_{\mathbb{T}} \int_{x_{0}}^{x} \phi(y, t) d y d x .
$$

Copyright (c) by SIAM. Unauthorized reproduction of this article is prohibited. 
We make the observation that $\partial_{x}$ and $\partial_{x}^{-1}$ generally do not commute since $\left[\partial_{x}, \partial_{x}^{-1}\right] \phi=$ $\int_{\mathbb{T}} \phi d x$, where $[P, Q]=P Q-Q P$. Consider $(2.11)$, written in the form

$$
\partial_{x}\left(\partial_{t} u+u \partial_{x} u\right)=\frac{n}{n-1}\left(\partial_{x} u\right)^{2}+f(t)
$$

with $n>1$. As a result of (2.13) and the fact that $\partial_{x}^{-1} \partial_{x} \phi=\phi-\int_{\mathbb{T}} \phi d x$, we may write $(2.15)$ in a nonlocal form as

$$
\partial_{x}\left(\partial_{t} u+u \partial_{x} u\right)=\frac{n}{n-1} \partial_{x}^{-1} \partial_{x}\left(\left(\partial_{x} u\right)^{2}\right)
$$

and then, using the periodicity of $u$, we obtain

$$
\partial_{t} u+u \partial_{x} u=\frac{n}{n-1} \partial_{x}^{-2} \partial_{x}\left(\left(\partial_{x} u\right)^{2}\right)
$$

Okamoto and Zhu [14] previously established local existence for (2.10) with $u \in H^{2}$, using a method introduced by Kato and Lai. Their approach requires showing uniqueness separately and then using uniqueness to prove continuous dependence on initial data. Here we instead derive a local well-posedness result in $C^{1}$ which follows from Picard iteration after rewriting the equation as an ordinary differential equation on an infinite-dimensional Banach space. This method can also be used to prove wellposedness in Sobolev spaces $H^{s}(\mathbb{T})$ for $s>3 / 2$ (see [10] and [12], for example, for a similar result for the Camassa-Holm equation).

3. Local existence of classical solutions. In [1], Arnold observed that the initial value problem for the classical Euler equations of a perfect fluid can be stated as a geometric problem of finding geodesics on the group of volume preserving diffeomorphisms. Following this observation, Ebin and Marsden [8] developed the functional analytic tools to establish sharp local well-posedness results for the Euler equations. This method has since been used for other equations with similar geometric interpretations; for example, Misiołek [12] obtained local well-posedness in $C^{1}(\mathbb{T})$ for the Camassa-Holm equation, which is the equation for geodesics of the $H^{1}$ metric on the Virasoro group.

In this section we develop an appropriate analytic framework for (2.17), using a similar approach to prove the following theorem.

THEOREM 3.1. Suppose that $n>1$. Then there exists a unique solution

$$
u \in C^{0}\left([0, T), C^{1}(\mathbb{T})\right) \cap C^{1}\left([0, T), C^{0}(\mathbb{T})\right)
$$

to the Cauchy problem for (2.17) with initial data $u_{0} \in C^{1}(\mathbb{T})$ for some $T>0$, and the solution depends continuously on the initial data.

Let $\gamma$ be the flow generated by $u$, that is,

$$
\frac{d \gamma}{d t}(x, t)=u(\gamma(x, t), t),
$$

or $u=\dot{\gamma} \circ \gamma^{-1}$. Then we obtain the equation

$$
\ddot{\gamma}=\frac{n}{n-1} \partial_{x}^{-2} \partial_{x}\left(\left(\partial_{x}\left(\dot{\gamma} \circ \gamma^{-1}\right)\right)^{2}\right) \circ \gamma
$$

from (2.17). Therefore it is sufficient to prove that

$$
F(X, \gamma)=\frac{n}{n-1}\left(\partial_{x}^{-2} \partial_{x}\left(\left(\partial_{x}\left(X \circ \gamma^{-1}\right)\right)^{2}\right)\right) \circ \gamma
$$

Copyright (c) by SIAM. Unauthorized reproduction of this article is prohibited. 
defines a continuously differentiable vector field in a neighborhood of the identity on the topological group $\mathcal{D}$ of $C^{1}$ class diffeomorphisms. Then Theorem 3.1 follows by Picard iteration over Banach spaces.

We remark that the smooth dependence on initial data for (3.1) implies only continuous dependence on initial data for (2.17). The map $\gamma \rightarrow \gamma^{-1}$ is continuous but not locally Lipschitz [8], and this prevents obtaining more regularity for the initial data to solution map by this method. The question of whether the regularity of the solution map $u_{0} \rightarrow u(t)$ can be improved, or not, is open. It is known, for instance, that it is not possible to improve the regularity of this map for the Camassa-Holm equation in Sobolev spaces [11].

In the remainder of this section, $C_{\gamma}$ will represent a generic constant depending only on the $C^{1}$ norms of $\gamma$ and $\gamma^{-1}$.

Proof of Theorem 3.1. Let us denote by $P_{\gamma}$ the operator given by conjugation

$$
P_{\gamma}(\phi):=P\left(\phi \circ \gamma^{-1}\right) \circ \gamma
$$

for any $\gamma \in \mathcal{D}$ and pseudodifferential operator $P$. Using this notation we write

$$
F(X, \gamma)=\frac{n}{n-1}\left(\partial_{x}^{-2} \partial_{x}\right)_{\gamma}\left(\left(\partial_{x}\right)_{\gamma} X\right)^{2} .
$$

Next we compute the directional derivative $\partial_{\gamma} F_{(X, \gamma)}$ and prove that it is a bounded linear map.

Note that $\left(\partial_{x}^{-2} \partial_{x}\right) f=\partial_{x}^{-1}\left\{f-\int_{0}^{1} f d x\right\}$ is a bounded operator from $C^{0}(\mathbb{T})$ into $C^{1}(\mathbb{T})$. Furthermore we abuse the notation slightly and denote by $\mathcal{D}$ the connected component of orientation preserving $C^{1}$ diffeomorphisms of $\mathbb{T}$.

Let $s \rightarrow \gamma_{s}$ be a smooth curve in $\mathcal{D}$ such that $\gamma_{0}=i d$ and $\left.\partial_{s} \gamma_{s}\right|_{s=0}=W$ for $W \in C^{1}(\mathbb{T})$. Then we have

$$
\partial_{\gamma} F_{(X, \gamma)}(W)=\frac{n}{n-1}\left\{\left.\left(\partial_{\varepsilon} G_{\varepsilon}\right)\right|_{\varepsilon=0} \circ \gamma+W\left(\partial_{x} G_{\varepsilon}\right)_{\varepsilon=0} \circ \gamma\right\},
$$

where $G_{\varepsilon}=\partial_{x}^{-2} \partial_{x}\left\{\left(\partial_{x}\left(X \circ \gamma_{\varepsilon}^{-1}\right)\right)^{2}\right\}$. We know that $\partial_{x} \partial_{x}^{-1}$ gives the identity; hence the second summand on the right in (3.2) can be written as

$$
\left.W \partial_{x} G_{\varepsilon}\right|_{\varepsilon=0} \circ \gamma=\left\{\left(\left(\partial_{x}\right)_{\gamma} X\right)^{2}-\int_{0}^{1}\left(\partial_{x}\left(X \circ \gamma^{-1}\right)\right)^{2} d x\right\} W .
$$

Moreover, the computation of the first summand on the right in (3.2) is reduced by

$$
\left.\left(\partial_{\varepsilon} G_{\varepsilon}\right)\right|_{\varepsilon=0}=\partial_{x}^{-2} \partial_{x}\left(\left.\partial_{\varepsilon} H_{\varepsilon}\right|_{\varepsilon=0}\right)
$$

to determine $\left.\partial_{\varepsilon} H_{\varepsilon}\right|_{\varepsilon=0}$, where $H_{\varepsilon}=\left(\partial_{x}\left(X \circ \gamma_{\varepsilon}^{-1}\right)\right)^{2}$. Here a straightforward computation leads to

$$
\left.\partial_{\varepsilon} H_{\varepsilon}\right|_{\varepsilon=0}=-\left(\partial_{x}\left(X \circ \gamma^{-1}\right)\right)^{2} \partial_{x}\left(W \circ \gamma^{-1}\right)-\partial_{x}\left\{\left(W \circ \gamma^{-1}\right)\left(\partial_{x}\left(X \circ \gamma^{-1}\right)\right)^{2}\right\}
$$

Then, after an integration by parts, we obtain

$$
\begin{aligned}
\left.\partial_{\varepsilon} G_{\varepsilon}\right|_{\varepsilon=0}= & -\left(\partial_{x}\left(X \circ \gamma^{-1}\right)\right)^{2}\left(W \circ \gamma^{-1}\right)+\int_{0}^{1}\left(\partial_{x}\left(X \circ \gamma^{-1}\right)\right)^{2}\left(W \circ \gamma^{-1}\right) d x \\
& -\partial_{x}^{-2} \partial_{x}\left\{\left(\partial_{x}\left(X \circ \gamma^{-1}\right)\right)^{2} \partial_{x}\left(W \circ \gamma^{-1}\right)\right\} \\
& +\left.\left(x-\frac{1}{2}\right)\left\{\left(\partial_{x}\left(X \circ \gamma^{-1}\right)\right)^{2}\left(W \circ \gamma^{-1}\right)\right\}\right|_{0} ^{1} .
\end{aligned}
$$

Copyright $\odot$ by SIAM. Unauthorized reproduction of this article is prohibited. 
The last term on the left-hand side of the above inequality vanishes since $X$ and $W$ are periodic functions and $\gamma$ is an orientation preserving diffeomorphism. Therefore we have

$$
\begin{aligned}
\partial_{\gamma} F_{(X, \gamma)}(W)=\frac{n}{n-1}\{ & -\partial_{x}^{-2} \partial_{x}\left\{\left(\partial_{x}\left(X \circ \gamma^{-1}\right)\right)^{2} \partial_{x}\left(W \circ \gamma^{-1}\right)\right\} \circ \gamma \\
& +\int_{0}^{1}\left(\partial_{x}\left(X \circ \gamma^{-1}\right)\right)^{2} W \circ \gamma^{-1} d x \\
& \left.-W \int_{0}^{1}\left(\partial_{x}\left(X \circ \gamma^{-1}\right)\right)^{2} d x\right\} .
\end{aligned}
$$

The linearity of the map $W \rightarrow \partial_{\gamma} F_{(X, \gamma)}(W)$ is clear. Thus we proceed to show that it is bounded. It is sufficient to estimate the $C^{1}$ norms of all three summands on the right in (3.6). The second and third terms are both bounded by $C_{\gamma}\|W\|_{C^{0}}\|X\|_{C^{1}}^{2}$. For the first term on the right in (3.6), we have

$$
\begin{aligned}
\left\|\left(\partial_{x}^{-2} \partial_{x}\right)_{\gamma}\left\{\left(\left(\partial_{x}\right)_{\gamma} X\right)^{2}\left(\partial_{x}\right)_{\gamma} W\right\}\right\|_{C^{1}} \leq & \left\|\left(\partial_{x}^{-2} \partial_{x}\right)_{\gamma}\left\{\left(\left(\partial_{x}\right)_{\gamma} X\right)^{2}\left(\partial_{x}\right)_{\gamma} W\right\}\right\|_{C^{0}} \\
& +\left\|\left(\left(\partial_{x}\right)_{\gamma} X\right)^{2}\left(\partial_{x}\right)_{\gamma} W\right\|_{C^{0}}\|\gamma\|_{C^{1}}
\end{aligned}
$$

which is bounded by $C_{\gamma}\|X\|_{C^{1}}^{2}\|W\|_{C^{1}}$.

In the direction of $X$, the Gâteaux derivative of $F$ is given by

$$
\partial_{X} F_{(X, \gamma)}(W)=\frac{2 n}{n-1}\left(\partial_{x}^{-2} \partial_{x}\right)_{\gamma}\left(\left(\partial_{x}\right)_{\gamma} X\left(\partial_{x}\right)_{\gamma} W\right)
$$

and this is a bounded map since

$$
\begin{aligned}
\left\|\partial_{X} F_{(X, \gamma)}(W)\right\|_{C^{1}} \leq & C_{n}\left\|\left(\partial_{x}^{-2} \partial_{x}\right)_{\gamma}\left(\left(\partial_{x}\right)_{\gamma} X\left(\partial_{x}\right)_{\gamma} W\right)\right\|_{C^{0}} \\
& +\left\|\left(\partial_{x}\right)_{\gamma} X\left(\partial_{x}\right)_{\gamma} W-\int_{\mathbb{T}}\left(\partial_{x}\right)_{\gamma} X\left(\partial_{x}\right)_{\gamma} W d x\right\|_{C^{0}}\|\gamma\|_{C^{1}} \\
\leq & C_{n, \gamma}\|X\|_{C^{1}}\|W\|_{C^{1}},
\end{aligned}
$$

where $C_{n, \gamma}$ depends only on $n$ and $C^{1}$ norms of $\gamma$ and $\gamma^{-1}$.

In order to complete the proof of Theorem 3.1 it is sufficient to show that $F$ is Fréchet differentiable; i.e., both directional derivatives $\partial_{\gamma} F$ and $\partial_{X} F$ are continuous maps.

Continuity of $(X, \gamma) \rightarrow \partial_{\gamma} F_{(X, \gamma)}(W)$. The following inequality reduces the proof of continuity of $\partial_{\gamma} F_{(X, \gamma)}(W)$ to estimating the two summands on the right-hand side:

$$
\begin{aligned}
\left\|\partial_{\gamma} F_{\left(X_{1}, \gamma_{1}\right)}(W)-\partial_{\gamma} F_{\left(X_{2}, \gamma_{2}\right)}(W)\right\|_{C^{1}} \leq & \left\|\partial_{\gamma} F_{\left(X_{1}, \gamma_{1}\right)}(W)-\partial_{\gamma} F_{\left(X_{2}, \gamma_{1}\right)}(W)\right\|_{C^{1}} \\
& +\left\|\partial_{\gamma} F_{\left(X_{2}, \gamma_{1}\right)}(W)-\partial_{\gamma} F_{\left(X_{2}, \gamma_{2}\right)}(W)\right\|_{C^{1}}
\end{aligned}
$$

where the inequality holds up to a constant depending on $n$. We rewrite the $C^{1}$ norm that we wish to estimate to show continuity in $X$ as

Copyright $@$ by SIAM. Unauthorized reproduction of this article is prohibited. 


$$
\begin{aligned}
\left\|\partial_{\gamma} F_{\left(X_{1}, \gamma\right)}-\partial_{\gamma} F_{\left(X_{2}, \gamma\right)}\right\|_{C^{1}} \leq & \left\|\left(\partial_{x}^{-2} \partial_{x}\right)_{\gamma}\left\{\left(\left(\left(\partial_{x}\right)_{\gamma} X_{1}\right)^{2}-\left(\left(\partial_{x}\right)_{\gamma} X_{2}\right)^{2}\right)\left(\partial_{x}\right)_{\gamma} W\right\}\right\|_{C^{1}} \\
& +\left|\int_{0}^{1}\left(\partial_{x}\left(X_{1} \circ \gamma-X_{2} \circ \gamma\right)\right)^{2} W \circ \gamma^{-1} d x\right| \\
& +|W|\left|\int_{0}^{1}\left(\partial_{x}\left(X_{1} \circ \gamma-X_{2} \circ \gamma\right)\right)^{2} d x\right|
\end{aligned}
$$

The last two summands in (3.8) are bounded by $C_{\gamma}\left\|X_{1}-X_{2}\right\|_{C^{1}}^{2}\|W\|_{C^{0}}$. For the remaining term, we have

$$
\begin{aligned}
& \left\|\left(\partial_{x}^{-2} \partial_{x}\right)_{\gamma}\left\{\left(\left(\left(\partial_{x}\right)_{\gamma} X_{1}\right)^{2}-\left(\left(\partial_{x}\right)_{\gamma} X_{2}\right)^{2}\right)\left(\partial_{x}\right)_{\gamma} W\right\}\right\|_{C^{1}} \\
& \leq \quad\left\|\left(\partial_{x}^{-2} \partial_{x}\right)_{\gamma}\left\{\left(\left(\left(\partial_{x}\right)_{\gamma} X_{1}\right)^{2}-\left(\left(\partial_{x}\right)_{\gamma} X_{2}\right)^{2}\right)\left(\partial_{x}\right)_{\gamma} W\right\}\right\|_{C^{0}} \\
& \quad+\left\|\left(\left(\partial_{x}\left(X_{1} \circ \gamma^{-1}\right)\right)^{2}-\left(\partial_{x}\left(X_{2} \circ \gamma^{-1}\right)\right)^{2}\right) \partial_{x}\left(W \circ \gamma^{-1}\right)\right\|_{C^{0}}\left\|\partial_{x} \gamma\right\|_{C^{0}}
\end{aligned}
$$

which is bounded by $C_{\gamma}\left\|X_{1}-X_{2}\right\|_{C^{1}}\left\|X_{1}+X_{2}\right\|_{C^{1}}\|W\|_{C^{1}}$.

Our next estimate establishes continuity of $\gamma \rightarrow \partial_{\gamma} F_{(X, \gamma)}(W)$. Note that it is sufficient to consider

$$
\begin{aligned}
& \left\|\partial_{\gamma} F_{(X, \gamma)}(W)-\partial_{\gamma} F_{(X, i d)}(W)\right\|_{C^{1}} \\
& \leq\left\|\left(\partial_{x}^{-2} \partial_{x}\right)_{\gamma}\left\{\left(\left(\partial_{x}\right)_{\gamma} X\right)^{2}\left(\partial_{x}\right)_{\gamma} W\right\}-\partial_{x}^{-2} \partial_{x}\left(\left(\partial_{x} X\right)^{2} \partial_{x} W\right)\right\|_{C^{1}} \\
& \quad+\left|\int_{0}^{1}\left\{\left(\partial_{x}\left(X \circ \gamma^{-1}\right)\right)^{2}\left(W \circ \gamma^{-1}\right)-\left(\partial_{x} X\right)^{2} W\right\} d x\right| \\
& \quad+\|W\|_{C^{0}}\left|\int_{0}^{1}\left\{\left(\partial_{x}\left(X \circ \gamma^{-1}\right)\right)^{2}-\left(\partial_{x} X\right)^{2}\right\} d x\right|
\end{aligned}
$$

where the inequality is up to a constant depending on $n$. After adding and subtracting the appropriate terms, (3.10) is bounded by

$$
\|W\|_{C^{0}}\left\|\partial_{x} X\right\|_{C^{0}}\left\|\left(\partial_{x} X \circ \gamma^{-1}\right) \partial_{x} \gamma^{-1}-\partial_{x} X\right\|_{C^{0}}+\left\|\partial_{x} X\right\|_{C^{0}}^{2}\|W\|_{C^{1}}\|\gamma-i d\|_{C^{0}}
$$

which is bounded (up to a constant $C_{\gamma}$ ) by $\|X\|_{C^{1}}^{2}\|W\|_{C^{1}}\|\gamma-i d\|_{C^{1}}$. The term in (3.11) is estimated similarly. Hence in order to establish continuity in $\gamma$ it is sufficient to bound

$$
\begin{aligned}
& \quad\left\|\left(\partial_{x}^{-2} \partial_{x}\right)_{\gamma}\left\{\left(\left(\partial_{x}\right)_{\gamma} X\right)^{2}\left(\partial_{x}\right)_{\gamma} W\right\}-\partial_{x}^{-2} \partial_{x}\left(\left(\partial_{x} X\right)^{2} \partial_{x} W\right)\right\|_{C^{1}} \\
& \leq\left\|\left(\partial_{x}^{-2} \partial_{x}\right)_{\gamma}\left\{\left(\left(\partial_{x}\right)_{\gamma} X\right)^{2}\left(\partial_{x}\right)_{\gamma} W\right\}-\partial_{x}^{-2} \partial_{x}\left(\left(\partial_{x} X\right)^{2} \partial_{x} W\right)\right\|_{C^{0}} \\
& +\|\left\{\left(\partial_{x}\left(X \circ \gamma^{-1}\right)^{2} \partial_{x}\left(W \circ \gamma^{-1}\right)\right\} \circ \gamma \partial_{x} \gamma-\left(\partial_{x} X\right)^{2} \partial_{x} W \|_{C^{0}}\right.
\end{aligned}
$$

The norm in (3.14) is equal to

$$
\left\|\partial_{x} W\left(\partial_{x} X\right)^{2}\left\{\left(\partial_{x} \gamma^{-1}\right)^{2} \circ \gamma-1\right\}\right\|_{C_{0}},
$$

which is bounded by $C_{\gamma}\left\|\partial_{x} X\right\|_{C^{0}}^{2}\left\|\partial_{x} W\right\|_{C^{0}}\|\gamma-i d\|_{C^{1}}$. For (3.13) it is sufficient to estimate

$$
\left\|\left(\partial_{x}^{-2} \partial_{x}\right)_{\gamma} S-\partial_{x}^{-2} \partial_{x} S\right\|_{C^{0}}+\left\|\partial_{x}^{-2} \partial_{x} S-\partial_{x}^{-2} \partial_{x}\left(\left(\partial_{x} X\right)^{2} \partial_{x} W\right)\right\|_{C^{0}}
$$

Copyright (c) by SIAM. Unauthorized reproduction of this article is prohibited. 
where

$$
S=S(X, \gamma, W)=\left(\partial_{x}\left(X \circ \gamma^{-1}\right)\right)^{2} \circ \gamma \partial_{x}\left(W \circ \gamma^{-1}\right) \circ \gamma .
$$

After a change of variables we regroup the terms in the first summand of (3.16) to obtain

$$
\begin{aligned}
\left(\partial_{x}^{-2} \partial_{x}\right)_{\gamma} S-\partial_{x}^{-2} \partial_{x} S & =\int_{0}^{1} \int_{\gamma^{-1}(x)}^{x} S(y) \partial_{x} \gamma(y) d y d x+\int_{x_{0}}^{x} S(y)\left(\partial_{x} \gamma(y)-1\right) d y \\
& -\int_{0}^{1} \int_{x_{0}}^{x} S(y)\left(\partial_{x} \gamma(y)-1\right) d y d x \\
- & -\left(\gamma(x)-\frac{1}{2}\right) \int_{0}^{1} S \circ \gamma^{-1}(y) d y+\left(x-\frac{1}{2}\right) \int_{0}^{1} S(y) d y .
\end{aligned}
$$

The right-hand side of this equality can be simplified further as

$$
\begin{aligned}
& \left(\partial_{x}^{-2} \partial_{x}\right)_{\gamma} S-\partial_{x}^{-2} \partial_{x} S=\int_{0}^{1} \int_{\gamma^{-1}(x)}^{x} S(y) \partial_{x} \gamma(y) d y d x \\
& +\partial_{x}^{-2} \partial_{x}\left(S\left(\partial_{x} \gamma-1\right)\right)-(\gamma(x)-x) \int_{0}^{1} S \circ \gamma^{-1}(y) d y .
\end{aligned}
$$

Clearly the sup norms of all three summands in (3.20)-(3.21) vanish in the limit as $\gamma$ goes to $i d$ in $C^{1}$. The second summand in (3.16) is equal to the $C^{0}$ norm of

$$
\partial_{x}^{-2} \partial_{x}\left(\partial_{x} W\left(\partial_{x} X\right)^{2}\left\{\left(\partial_{x} \gamma^{-1}\right)^{3} \circ \gamma-1\right\}\right)
$$

which is bounded by $C_{\gamma}\|W\|_{C^{1}}\|X\|_{C^{1}}^{2}\|\gamma-i d\|_{C^{1}}$. Hence the continuity of $\gamma \rightarrow \partial F_{(X, \gamma)}$ follows.

The continuity of $(X, \gamma) \rightarrow \partial_{X} F_{(X, \gamma)}$ can be shown analogously. Therefore $F(X, \gamma)$ defines a continuously differentiable map in a neighborhood of $\left(i d, u_{0}\right)$. This completes the proof of Theorem 3.1.

4. Global existence for $\boldsymbol{n} \geq 3$. In this section we investigate the persistence of solutions of the initial value problem for (2.17) and show that, unlike the twodimensional case where solutions may blow up in finite time (see [3], [5]), they persist for $n \geq 3$ in the appropriate function spaces.

For the theorem below let us use the following notation:

$$
X_{n}(\mathbb{T})= \begin{cases}W^{2, \infty}(\mathbb{T}), & n=3, \\ W^{2, \frac{n-1}{n-3}}(\mathbb{T}), & n>3,\end{cases}
$$

and

$$
Y_{n}(\mathbb{T})= \begin{cases}W^{1, \infty}(\mathbb{T}), & n=3, \\ W^{1, \frac{n-1}{n-3}}(\mathbb{T}), & n>3\end{cases}
$$

Copyright $@$ by SIAM. Unauthorized reproduction of this article is prohibited. 
TheOREM 4.1. Let $n \geq 3$ and assume that $u_{0}(x) \in X_{n}(\mathbb{T})$. Then the solution $u(x, t)$ from Theorem 3.1 has a unique extension to all $T<\infty$ such that $u(x, t) \in$ $C^{0}\left([0, T], X_{n}(\mathbb{T})\right) \cap C^{1}\left([0, T], Y_{n}(\mathbb{T})\right)$.

Proof. Consider (2.10), expressed in the form

$$
\partial_{t} \partial_{x}^{2} u+u \partial_{x}^{3} u+\frac{n-3}{n-1} \partial_{x} u \partial_{x}^{2} u=0 .
$$

Using the flow $\gamma$ of $u(\dot{\gamma}=u \circ \gamma)$ in (4.1), we first solve for $\partial_{x}^{2} u$ :

$$
\partial_{x}^{2} u \circ \gamma(t)=u_{0}^{\prime \prime} \exp \left(-\frac{n-3}{n-1} \int_{0}^{t} \partial_{x} u \circ \gamma(s) d s\right) .
$$

By the identity $\partial_{x} \dot{\gamma}=\partial_{x} u \circ \gamma \partial_{x} \gamma$, we also have

$$
\partial_{x} \gamma(t)=\exp \left(\int_{0}^{t} \partial_{x} u \circ \gamma(s) d s\right) .
$$

Therefore

$$
\partial_{x}^{2} u \circ \gamma(t)\left(\partial_{x} \gamma(t)\right)^{\frac{n-3}{n-1}}=u_{0}^{\prime \prime} .
$$

Note that Theorem 3.1 implies that $\gamma \in C^{1}$ locally in time and it follows, given $\partial_{x} \gamma(0)=1$, that there exists an interval, $t \in[0, \tau(\varepsilon))$, over which $0<\varepsilon \leq \inf _{\mathrm{x} \in \mathbb{T}}$ $\partial_{\mathrm{x}} \gamma(\mathrm{t}) \leq \sup _{\mathrm{x} \in \mathbb{T}} \partial_{\mathrm{x}} \gamma(\mathrm{t}) \leq \varepsilon^{-1}$. Equation (4.4) then implies that, locally, $u \in X_{n}(\mathbb{T})$, since $\gamma$ maps $\mathbb{T}$ diffeomorphically to itself. In turn, (2.16) shows that $\partial_{t} u \in Y_{n}(\mathbb{T})$ over the same time interval. (With additional assumptions on the data, further regularity can also be bootstrapped to higher derivatives.)

Assuming then that sufficient smoothness holds locally in time, we find on multiplying (4.1) by $\left|\partial_{x}^{2} u\right|^{p-2} \partial_{x}^{2} u$ that

$$
\partial_{t}\left|\partial_{x}^{2} u\right|^{p}+u \partial_{x}\left|\partial_{x}^{2} u\right|^{p}+p \frac{n-3}{n-1} \partial_{x} u\left|\partial_{x}^{2} u\right|^{p}=0 .
$$

Since (2.2) and (2.8) imply that both $u$ and $\partial_{x} u$ are periodic functions of $x$, the same is true of $\partial_{x}^{2} u$, by (2.11). One therefore obtains, on integrating (4.5) over $\mathbb{T}$,

$$
\frac{d}{d t} \int_{\mathbb{T}}\left|\partial_{x}^{2} u\right|^{p} d x+\left(p \frac{n-3}{n-1}-1\right) \int_{\mathbb{T}} \partial_{x} u\left|\partial_{x}^{2} u\right|^{p} d x=0,
$$

from which it follows that the $L^{\frac{n-1}{n-3}}(\mathbb{T})$ norm of $\partial_{x}^{2} u$ is uniformly conserved in time for $n>3$. The case $n=3$ can either be considered as the limit $n \rightarrow 3$ with $p \rightarrow \infty$ in (4.6), or directly using (4.1) which shows that $\partial_{x}^{2} u$ is constant along characteristics and hence its $L^{\infty}(\mathbb{T})$ norm is uniformly conserved.

Periodicity of $u(x, t)$ in $x$ implies there exists a zero for $\partial_{x} u$, say at $x=x_{0}(t)$, and so for $x, x_{0} \in \mathbb{T}$,

$$
\partial_{x} u(x, t)=\int_{x_{0}}^{x} \partial_{y}^{2} u(y, t) d y
$$

For $n>3$, we therefore have the estimate

$$
\left|\partial_{x} u(x, t)\right| \leq\left.\left|x-x_{0}\right|^{\frac{2}{n-1}}|| u_{0}^{\prime \prime}\right|_{\frac{n-1}{n-3}} \leq\left\|u_{0}^{\prime \prime}\right\|_{\frac{n-1}{n-3}}
$$

Copyright $@$ by SIAM. Unauthorized reproduction of this article is prohibited. 
using Hölder's inequality, and so

$$
\left\|\partial_{x} u\right\|_{\infty} \leq\left\|u_{0}^{\prime \prime}\right\|_{\frac{n-1}{n-3}} .
$$

If $n=3$, then

$$
\left\|\partial_{x}^{2} u\right\|_{\infty}=\left\|u_{0}^{\prime \prime}\right\|_{\infty}
$$

which means

$$
\left|\partial_{x} u(x, t)\right| \leq\left|x-x_{0}\right|\left\|u_{0}^{\prime \prime}\right\|_{\infty} \leq\left\|u_{0}^{\prime \prime}\right\|_{\infty}
$$

for all $x \in \mathbb{T}$, and so

$$
\left\|\partial_{x} u\right\|_{\infty} \leq\left\|u_{0}^{\prime \prime}\right\|_{\infty}
$$

for all $t>0$.

Further, since $u(x, t)-u_{0}(x)$ has mean zero by (2.14), there exists $x=x_{1}(t)$, where $u\left(x_{1}, t\right)=u_{0}\left(x_{1}\right)$ and, for $x, x_{1} \in \mathbb{T}$, we have

$$
u(x, t)=u_{0}(x)+\int_{x_{1}}^{x}\left(\partial_{y} u(y, t)-u_{0}^{\prime}(y)\right) d y .
$$

It follows that

$$
|u(x, t)| \leq\left\|u_{0}\right\|_{\infty}+\left|x-x_{1}\right|\left(\left\|\partial_{x} u\right\|_{\infty}+\left\|u_{0}^{\prime}\right\|_{\infty}\right)
$$

for all $x \in \mathbb{T}$, which gives the inequality

$$
\|u\|_{\infty} \leq\left\|\partial_{x} u\right\|_{\infty}+\left\|u_{0}\right\|_{C^{1}} .
$$

Combining the results of the previous two paragraphs shows that

$$
\|u\|_{C^{1}} \leq\left\|u_{0}\right\|_{C^{2}} \quad \text { for } \quad n=3,
$$

and

$$
\|u\|_{C^{1}} \leq\left\|u_{0}\right\|_{C^{1}}+\|\left. u_{0}^{\prime \prime}\right|_{\frac{n-1}{n-3}} \quad \text { for } \quad n>3 .
$$

Finally, on using the properties of the operators $\partial_{x}^{-1} \partial_{x}$ and $\partial_{x}^{-2} \partial_{x}$ in (2.16) and (2.17) together with the above estimates, it is seen that $\left\|\partial_{t} u\right\|_{\infty}$ and $\left\|\partial_{t} \partial_{x} u\right\|_{\infty}$ are majorized by a function of $\left\|u_{0}\right\|_{C^{2}}$ for $n=3$, while $\left\|\partial_{t} u\right\|_{\infty}$ and $\left\|\partial_{t} \partial_{x} u\right\|_{\frac{n-1}{n-3}}$ are majorized by a function of $\left\|u_{0}\right\|_{W^{2, \frac{n-1}{n-3}}}$ for $n>3$.

In both of these cases it follows that the $C^{1}(\mathbb{T})$ norm of $u$ and the $C^{0}(\mathbb{T})$ norm of $\partial_{t} u$ remain uniformly bounded in time over any interval of local existence and, by bootstrapping the arguments of Theorem 3.1, the solution can be continued, globally, in time.

As a remark, we note here how a blow up argument made in [3], which involves a nontrivial class of separable solutions to (2.10) for $n=2$, fails to apply in the case $n>3$. In particular, the possible appearance of a $(\tau-t)^{-1}$ factor, $\tau>0$, in the two-dimensional case no longer exists in higher dimensions.

Given the solution form $u(x, t)=X(x) T(t),(2.10)$ reduces to

$$
\lambda X^{\prime \prime}(x)+\frac{n-3}{n-1} X^{\prime}(x) X^{\prime \prime}(x)+X(x) X^{\prime \prime \prime}(x)=0, x \in \mathbb{T},
$$

Copyright $@$ by SIAM. Unauthorized reproduction of this article is prohibited. 
where

$$
\dot{T}(t)-\lambda T(t)^{2}=0, t \geq 0,
$$

and $\lambda$ is a constant. Multiplying (4.9) by $\left|X^{\prime \prime}(x)\right|^{\frac{5-n}{n-3}} X^{\prime \prime}(x)$ now gives

$$
\lambda\left|X^{\prime \prime}(x)\right|^{\frac{n-1}{n-3}}+\frac{n-3}{n-1}\left(X^{\prime}(x)\left|X^{\prime \prime}(x)\right|^{\frac{n-1}{n-3}}+X(x)\left(\left|X^{\prime \prime}(x)\right|^{\frac{n-1}{n-3}}\right)^{\prime}\right)=0 .
$$

By using periodicity, an integration of (4.11) over $\mathbb{T}$ for $n>3$ therefore shows

$$
\lambda \int_{\mathbb{T}}\left|X^{\prime \prime}(x)\right|^{\frac{n-1}{n-3}} d x=0
$$

and the result then follows. We note that there are in general one or more points of inflection in nontrivial, periodic solutions, which prevents this argument from holding in two dimensions.

5. Weak solutions. In this section, we construct a basic, piecewise differentiable class of weak solutions $u(x, t) \in C^{0}\left([0, T), P C^{1}(\mathbb{T})\right) \cap C^{1}\left([0, T), P C^{0}(\mathbb{T})\right)$ to $(2.11)$, which are found to exist for all $T>0$, regardless of the underlying dimension.

For every vector field $\Phi(\mathbf{x}, t) \in C_{0}^{\infty}\left([0, T) \times \mathbb{T} \times \mathbb{R}^{n-1} ; \mathbb{R}^{n}\right)$ such that $\nabla \cdot \Phi=0$, and for every scalar function $\theta(\mathbf{x}, t) \in C_{0}^{\infty}\left([0, T) \times \mathbb{T} \times \mathbb{R}^{n-1} ; \mathbb{R}\right)$, the velocity field $\mathbf{u}(\mathbf{x}, t)$ in $(2.1 \mathrm{~b})$ satisfies

$$
\begin{gathered}
\int_{Q} \partial_{t} \Phi \cdot \mathbf{u}+(\nabla \Phi \mathbf{u}) \cdot \mathbf{u} d \mathbf{x} d t+\int_{\mathbb{T} \times \mathbb{R}^{n-1}} \Phi(\mathbf{x}, 0) \cdot \mathbf{u}(\mathbf{x}, 0) d \mathbf{x}=0, \\
\int_{\mathbb{R}^{n}} \nabla \theta \cdot \mathbf{u} d \mathbf{x}=0,
\end{gathered}
$$

where $Q=[0, T) \times \mathbb{T} \times \mathbb{R}^{n-1}$.

In terms of (2.2), (5.1) and (5.2) reduce to

$$
\begin{array}{r}
\int_{Q} \partial_{t} \phi u-\partial_{t} \Phi^{\prime} \cdot \mathbf{v} \partial_{x} u d \mathbf{x} d t \\
+\int_{Q} \partial_{x} \phi u^{2}-\left(\partial_{x} \Phi^{\prime}+\nabla^{\prime} \phi\right) \cdot \mathbf{v} u \partial_{x} u+\left(\nabla^{\prime} \Phi^{\prime} \mathbf{v}\right) \cdot \mathbf{v}\left(\partial_{x} u\right)^{2} d \mathbf{x} d t \\
+\int_{\mathbb{T} \times \mathbb{R}^{n-1}} \phi(\mathbf{x}, 0) u(x, 0)-\Phi^{\prime}(\mathbf{x}, 0) \cdot \mathbf{v}(\mathbf{x}, 0) \partial_{x} u(x, 0) d \mathbf{x}=0, \\
\int_{\mathbb{T} \times \mathbb{R}^{n-1}} \partial_{x} \theta u-\nabla^{\prime} \theta \cdot \mathbf{v} \partial_{x} u d \mathbf{x}=0,
\end{array}
$$

in which we have used the notation $\Phi=\left(\phi, \Phi^{\prime}\right)$ to distinguish the first component from the remaining $n-1$ components of $\Phi$. Denoting by $[\mathbf{u}]=\mathbf{u}_{+}-\mathbf{u}_{-}$the jump in $\mathbf{u}$ across any smooth surface of discontinuity, $S$, and considering test functions whose support crosses $S,(5.2)$ shows that

$$
[\mathbf{u}] \cdot \mathbf{n}=0
$$

Copyright $@$ by SIAM. Unauthorized reproduction of this article is prohibited. 
where $\mathbf{n}=\left(\mathfrak{n}, \mathbf{n}^{\prime}\right)$ is normal to $S$. Then, by (5.4), we have

$$
[u] \mathfrak{n}-\left[\partial_{x} u\right] \mathbf{v} \cdot \mathbf{n}^{\prime}=0, \text { where } \mathbf{v}=\frac{1}{n-1} \mathbf{x}^{\prime} .
$$

In examining weak, frontlike, piecewise continuous solutions for which $[u]=0$ and $\left[\partial_{x} u\right] \neq 0$ (see [6]), it follows that these discontinuities propagate so that $\mathbf{n}^{\prime} \cdot \mathbf{x}^{\prime}=$ 0 . A weak formulation specific to such discontinuities may be derived by means of appropriate choice of test functions from (5.3), or by observing that (2.11) may be written in conservation form as

$$
\partial_{t}\left(\left(\partial_{x} u\right)^{1-n}\right)+\partial_{x}\left(u\left(\partial_{x} u\right)^{1-n}\right)+(n-1)\left(\partial_{x} u\right)^{-n} f=0, x \in \mathbb{T} .
$$

We will admit weak solutions, $u(x, t)$, which satisfy the relation

$$
\begin{array}{r}
\int_{\mathfrak{Q}} \partial_{t} \varphi\left(\partial_{x} u\right)^{1-n}+\partial_{x} \varphi u\left(\partial_{x} u\right)^{1-n}-(n-1) f \varphi\left(\partial_{x} u\right)^{-n} d x d t \\
+\int_{\mathbb{T}} \varphi(x, 0)\left(\partial_{x} u\right)^{1-n}(x, 0) d x=0
\end{array}
$$

for all $\varphi(x, t) \in C_{0}^{\infty}(\mathfrak{Q})$, where $\mathfrak{Q}=[0, T) \times \mathbb{T}$. Using standard Rankine-Hugoniottype arguments [6], discontinuities in $\partial_{x} u$ that jump across a curve $x=\psi(t)$ are seen to satisfy

$$
(-\dot{\psi}+u(\psi, t))\left[\left(\partial_{x} u\right)^{1-n}\right]=0,
$$

and such discontinuities therefore propagate with the flow of $(2.11)$; i.e., $\psi(t)$ is a member of the characteristic family $\dot{\gamma}=u \circ \gamma$.

5.1. Piecewise affine solutions. We begin by commenting on the general case of periodic, $N$-phase, piecewise affine solutions. Given that both $\partial_{x} u$ and $\partial_{t} u$ may be discontinuous across the curves $x=\psi_{i}(t), 1 \leq i \leq N-1$, in order for $u$ to remain continuous there we must have $[u](\gamma(t), t)=0$, and so $\frac{d}{d t}[u](\gamma(t), t)=0$. As a result, $\partial_{t}[u]+u \partial_{x}[u]=0$, and first derivative jumps are seen to satisfy the relations

$$
\left[\partial_{t} u\right]+u\left[\partial_{x} u\right]=0 \text { and }\left[\partial_{x} p\right]=0
$$

from (2.3). Under these conditions on $u$, the expression for $f(t)$, which was obtained in (2.13) by integrating $(2.11)$ for $u \in C^{1}(\mathbb{T})$, remains unchanged:

$$
f=-\frac{n}{n-1} \int_{\mathbb{T}}\left(\partial_{x} u\right)^{2} d x .
$$

In the special case, $N=2$, which we consider here, our form of solution is given by the periodic extension of the function

$$
u(x, t)=\alpha+ \begin{cases}x p, & x \in(0, \tilde{\phi}), \\ \tilde{\phi} p+(x-\tilde{\phi}) q, & x \in(\tilde{\phi}, \tilde{\psi}), \\ \tilde{\phi} p+(\tilde{\psi}-\tilde{\phi}) q+(x-\tilde{\psi}) p, & x \in(\tilde{\psi}, 1),\end{cases}
$$

where $\tilde{\phi}=\phi-[[\phi]], \tilde{\psi}=\psi-[[\psi]]$, with [[.]] denoting the "integer part" of the argument. The functions $\tilde{\phi}(t)$ and $\tilde{\psi}(t)$ are the representatives in $[0,1]$ of the phase 
curves $\phi(t), \psi(t) \in(-\infty, \infty)$, which start out from $\phi(0), \psi(0) \in[0,1]$ and separate those regions where $\partial_{x} u(x, t)$ periodically takes on values of $p(t)$ or $q(t)$.

Proceeding heuristically for the moment, periodicity of $u(x, t)$ requires that

$$
\mathcal{N}(t)=\phi(t) p(t)+(\psi(t)-\phi(t)) q(t)+(1-\psi(t)) p(t)=0 .
$$

Given the spatial periodicity in pressure (see (2.9)), we recall that integration of (2.3) over one period showed the integral $\int_{0}^{1} u(x, t) d x$ to be independent of time. This allows (5.13) to be used to give an expression for $\alpha(t)$. The result may be written, for instance, in terms of $p, \tilde{\phi}$, and $\tilde{\psi}$, as

$$
\alpha+\frac{p}{2}(\tilde{\phi}+\tilde{\psi}-1)=c
$$

where we have set $c=\int_{0}^{1} u(x, 0) d x=\alpha(0)+\frac{p(0)-q(0)}{2}(\psi(0)-\phi(0))(\phi(0)+\psi(0)-1)$. We will assume that $0<\phi(0)<\psi(0)<1$. Choosing, for convenience, $c=0$, the "average characteristic" must propagate with speed zero and we will see, consequently, that both $\phi(t)$ and $\psi(t)$ remain in $[0,1]$ for all time. The distinctions between $\tilde{\phi}$ and $\phi, \tilde{\psi}$, and $\psi$ will therefore not be made further here.

Combining (2.11), (5.12), and (5.13) now gives

$$
\dot{p}=\frac{1}{n-1} p^{2}+f, \quad \dot{q}=\frac{1}{n-1} q^{2}+f,
$$

where

$$
f=-\frac{n}{n-1}\left(\phi p^{2}+(\psi-\phi) q^{2}+(1-\psi) p^{2}\right)
$$

Also, by (5.10),

$$
\dot{\phi}=\alpha+\phi p
$$

and

$$
\dot{\psi}=\alpha+\phi p+(\psi-\phi) q .
$$

We next verify that (5.14) follows from the system of equations (5.16)-(5.19). Differentiation and some simplification gives

$$
\begin{gathered}
\dot{\mathcal{N}}=-(\psi-\phi)(p-q) q+(\psi-\phi) q^{2}+(\phi+(1-\psi))\left(\frac{p^{2}}{n-1}+f\right)+(\psi-\phi)\left(\frac{q^{2}}{n-1}+f\right) \\
=-(\psi-\phi)(p-q) q-\left((\phi+(1-\psi)) p^{2}+(\psi-\phi) q^{2}\right) \\
=-p(q(\psi-\phi)+p(\phi+(1-\psi))) \\
=-p \mathcal{N},
\end{gathered}
$$

and so $\mathcal{N}(t)=\mathcal{N}(0) e^{-\int_{0}^{t} p(s) d s}$. In particular, taking periodic data, $\mathcal{N}(0)=0$, means that $\mathcal{N}(t)=0, t>0$. We may therefore use (5.14) to write (5.19) as

$$
(1-\psi)^{\circ}=-\alpha+(1-\psi) p
$$

Copyright $@$ by SIAM. Unauthorized reproduction of this article is prohibited. 
and again employ (5.14) to express the following phase fractions as functions of $p$ and $q$ :

$$
\psi-\phi=\frac{p}{p-q}, \quad \phi+(1-\psi)=\frac{-q}{p-q} .
$$

Using these relations in (5.17) leads to

$$
f=\frac{n}{n-1} p q
$$

from which (5.16) reduces to the autonomous system

$$
\begin{gathered}
\dot{p}=\frac{1}{n-1}\left(p^{2}+n p q\right), \\
\dot{q}=\frac{1}{n-1}\left(q^{2}+n p q\right) .
\end{gathered}
$$

Subtracting (5.18) from (5.19) implies

$$
\psi(t)-\phi(t)=(\psi(0)-\phi(0)) \exp \left(\int_{0}^{t} q(s) d s\right),
$$

which means that the center phase fraction does not collapse as long as $\int_{0}^{t} q(s) d s$ remains bounded away from $-\infty$. Similarly, adding (5.18) and (5.20) gives

$$
\phi(t)+(1-\psi(t))=\left(\phi(0)+(1-\psi(0)) \exp \left(\int_{0}^{t} p(s) d s\right)\right.
$$

and the outer phase fraction exists as long as $\int_{0}^{t} p(s) d s>-\infty$. Comparing (5.25) and (5.26) shows also that periodicity imposes the following requirement on $p(t)$ and $q(t)$ in terms of their initial phase fractions:

$$
(5.27)\left(\phi(0)+(1-\psi(0)) \exp \left(\int_{0}^{t} p(s) d s\right)+(\psi(0)-\phi(0)) \exp \left(\int_{0}^{t} q(s) d s\right)=1 .\right.
$$

Using (5.16) to compute $p-q$ next gives

$$
p(t)-q(t)=(p(0)-q(0)) \exp \left(\frac{1}{n-1} \int_{0}^{t} p(s)+q(s) d s\right),
$$

which shows $p(t)-q(t)$ cannot change sign. By (5.21), $p(t)$ and $q(t)$ consequently keep their signs as long as neither phase fraction collapses. This can alternatively be seen by considering a sketch of $u$ and observing that $p$ and $q$ have opposite signs and can vanish only simultaneously. Thus, without loss of generality, we subsequently assume $p(t)>0>q(t)$, for at least some $t \geq 0$.

In the following lemma, we solve the system (5.23), (5.24) implicitly in order to show that two-phase solutions of the type (5.13) exist for all time.

LEMMA 5.1. Let $(p(t), q(t))$ satisfy (5.23), (5.24) with initial data $p(0)>0>q(0)$ (respectively, $p(0)<0<q(0)$ ). Then $(p(t), q(t))$ exists for all $t \in(-\infty, \infty)$ and satisfies $p(t)>0>q(t)$ (respectively, $p(t)<0<q(t)$ ). Further, $(p(t), q(t)) \rightarrow(0,0)$ as $t \rightarrow \pm \infty$, and $\|u(., t)\|_{C^{1}}+\left\|\partial_{t} u(., t)\right\|_{C^{0}} \rightarrow 0$ as $t \rightarrow \pm \infty$. 
Proof. Writing (5.23) and (5.24) using polar variables $p(t)=r(t) \cos \theta(t), q(t)=$ $r(t) \sin \theta(t), r(t) \geq 0$, leads to the following:

$$
\dot{r}(t)=\frac{r^{2}}{n-1}\left(\cos ^{3}(\theta(t))+n(\cos \theta(t)+\sin \theta(t)) \cos \theta(t) \sin \theta(t)+\sin ^{3}(\theta(t))\right),
$$

and

$$
\dot{\theta}(t)=r(\cos \theta(t)-\sin \theta(t)) \cos \theta(t) \sin \theta(t) .
$$

In the case $p(0)>0>q(0), \theta(0) \in(-\pi / 2,0)$, so by $(5.30) \dot{\theta}(0)<0$ and, as long as $\theta(t) \in(-\pi / 2,0), \dot{\theta}(t)<0$.

We will show that $\theta(t) \rightarrow-\pi / 2$ as $t \rightarrow \infty(\theta(t) \rightarrow 0$ as $t \rightarrow-\infty)$ by using (5.29) and (5.30). Integrating the resulting expression for $\frac{d \ln r}{d \theta}$ gives

$$
r(\theta)=c|\cos \theta \sin \theta|^{\frac{1}{n-1}}|\cos \theta-\sin \theta|^{-\frac{n+1}{n-1}},
$$

where $c>0$ denotes a generic constant. Inserting this expression for $r(\theta)$ in (5.30) results in

$$
\begin{aligned}
\dot{\theta}(t) & =c|\cos \theta \sin \theta|^{\frac{1}{n-1}}|\cos \theta-\sin \theta|^{-\frac{n+1}{n-1}}(\cos \theta-\sin \theta) \cos \theta \sin \theta \\
& =-c|\cos \theta \sin \theta|^{\frac{n}{n-1}}|\cos \theta-\sin \theta|^{-\frac{2}{n-1}}
\end{aligned}
$$

for $\theta(t) \in(-\pi / 2,0)$, and so

$$
\int_{\theta(0)}^{\theta(t)} \frac{|\cos \theta-\sin \theta|^{\frac{2}{n-1}}}{|\cos \theta \sin \theta|^{\frac{n}{n-1}}} d \theta=-c t,-\frac{\pi}{2}<\theta(0)<0, c>0 .
$$

Since $\frac{n}{n-1}>1$, the integral expression diverges, both as $\theta(t) \rightarrow-\pi / 2(t \rightarrow+\infty)$ and as $\theta(t) \rightarrow 0(t \rightarrow-\infty)$. Thus $\theta(t)$ and, from (5.31), $r(t)$, are bounded, continuous functions of time, with $\theta(t) \in(-\pi / 2,0)$. By (5.31) then, $r(t) \rightarrow 0$ as $t \rightarrow \pm \infty$, and, noting that from (5.14), (5.15),

$$
\alpha(t)=-\frac{p(t)-q(t)}{2}(\psi(t)-\phi(t))(\phi(0)+\psi(0)-1),
$$

it follows from (5.35) and (5.36) that

$$
p(t), q(t), \alpha(t), \text { and } u(x, t) \rightarrow 0 \text { as } t \rightarrow \pm \infty .
$$

The remaining conclusions are easily obtained.

Finally, we show that for $c=0$ the phases remain in the interval $[0,1]$ for all time.

TheOrem 5.1. Suppose $c=0$ and $0<\phi(0)<\psi(0)<1$. Then the phases $\phi, \psi$ stay in $[0,1]$. In particular

$$
\phi(t) \in\left[\phi(0), \frac{1}{2}(\phi(0)+\psi(0)) \text { and } \psi(t) \in\left(\frac{1}{2}(\phi(0)+\psi(0)), \psi(0)\right]\right.
$$

for all $t>0$. Further, the time asymptotic behavior satisfies

$$
\lim _{t \rightarrow \infty} \phi(t)=\frac{1}{2}(\phi(0)+\psi(0))=\lim _{t \rightarrow \infty} \psi(t) .
$$

Copyright $@$ by SIAM. Unauthorized reproduction of this article is prohibited. 
Proof. With (5.15) giving $\alpha$ (for $c=0$ ), substituting into (5.18) and (5.20) shows that, by $(5.26)$,

$$
\dot{\phi}(t)=(1-\psi(t))^{\cdot}=\frac{p}{2}(\phi+(1-\psi))=\frac{1}{2}(\phi(0)+(1-\psi(0))) p(t) \exp \left(\int_{0}^{t} p(s) d s\right),
$$

and the individual phases therefore satisfy

$$
\phi(t)=\phi(0)+\frac{1}{2}(\phi(0)+(1-\psi(0)))\left(\exp \left(\int_{0}^{t} p(s) d s\right)-1\right)
$$

and

$$
\psi(t)=\psi(0)-\frac{1}{2}(\phi(0)+(1-\psi(0)))\left(\exp \left(\int_{0}^{t} p(s) d s\right)-1\right) .
$$

Now we examine (5.27). Assuming $q<0<p$, the first term is monotonically increasing in time and must converge, for $0<\phi(0)<\psi(0)<1$, to a positive limit. The second term is positive but monotonically decreasing, so it may converge, as $t \rightarrow \infty$, either to a positive limit or to zero. Formally, setting $0<\int_{0}^{\infty} p(t) d t=L<\infty$ and $-\infty \leq \int_{0}^{\infty} q(t) d t=M<0,(5.27)$ and (5.28) imply

$$
(\phi(0)+(1-\psi(0))) e^{L}+(\psi(0)-\phi(0)) e^{M}=1
$$

and

$$
\lim _{t \rightarrow \infty}(p(t)-q(t))=(p(0)-q(0)) e^{\frac{L+M}{n+1}}
$$

If $M$ is finite and $p(0) \neq q(0)$, then (5.38), together with the necessity for $p(t)$ to approach zero as $t \rightarrow \infty$, means that $\lim _{t \rightarrow \infty} q(t) \neq 0$. However, this implies that $M=-\infty$, a contradiction. On the other hand, if $M=-\infty$, then, since $L<\infty$, (5.38) requires that $\lim _{t \rightarrow \infty} q(t)=0$, which is permitted. We conclude that, for smooth solutions $p(t), q(t)$ to exist with $p(0)>0>q(0)$, we require that $L=\int_{0}^{\infty} p(t) d t<\infty$ and $M=\int_{0}^{\infty} q(t) d t=-\infty$. Thus, by (5.37),

$$
e^{L}=\frac{1}{\phi(0)+1-\psi(0)}
$$

Writing (5.35) in the form

$$
\phi(t)=\frac{1}{2}(\phi(0)-(1-\psi(0)))+\frac{1}{2}(\phi(0)+(1-\psi(0))) \exp \int_{0}^{t} p(s) d s,
$$

it follows that

$$
\lim _{t \rightarrow \infty} \phi(t)=\frac{1}{2}(\phi(0)+\psi(0))
$$

and, similarly,

$$
\lim _{t \rightarrow \infty} \psi(t)=\frac{1}{2}(\phi(0)+\psi(0)) .
$$

By the monoticity in time of $\int_{0}^{t} p(s) d s$, therefore $\phi(t) \in\left[\phi(0), \frac{1}{2}(\phi(0)+\psi(0))\right.$ and $\psi(t) \in\left(\frac{1}{2}(\phi(0)+\psi(0)), \psi(0)\right]$ for all $t>0$.

Copyright (c) by SIAM. Unauthorized reproduction of this article is prohibited. 
Acknowledgment. The first author would like to thank Barbara Keyfitz, colleagues, and staff for their support at the Fields Institute, Toronto, where part of this work was done.

\section{REFERENCES}

[1] V. ARnold, Sur la géométrie différentielle des groupes de Lie de dimension infinie et ses applications à l'hydrodynamique des fluides parfaits, Ann. Inst. Fourier (Grenoble), 16 (1966), pp. 319-361.

[2] F. CAlogero, A solvable nonlinear wave equation, Stud. Appl. Math., 70 (1984), pp. 189-199.

[3] S. Childress, G. R. Ierley, E. A. Spiegel, and W. R. Young, Blow-up of unsteady twodimensional Euler and Navier-Stokes solutions having stagnation-point form, J. Fluid Mech., 203 (1989), pp. 1-22.

[4] P. Constantin, The Euler equations and nonlocal conservative Riccati equations, Int. Math. Res. Not., 9 (2000), pp. 455-465.

[5] S. M. Cox, Two-dimensional flow of a viscous fluid in a channel with porous walls, J. Fluid Mech., 227 (1991), pp. 1-33.

[6] C. M. Dafermos, Hyperbolic Conservation Laws in Continuum Physics, Grundlehren Math. Wiss. 325, Springer-Verlag, Berlin, 2000.

[7] D. G. Ebin, A concise presentation of the Euler equations of hydrodynamics, Comm. Partial Differential Equations, 9 (1984), pp. 539-559.

[8] D. G. Ebin And J. Marsden, Groups of diffeomorphisms and the motion of an incompressible fluid, Ann. of Math. (2), 92 (1970), pp. 102-163.

[9] J. Eells, A setting for global analysis, Bull. Amer. Math. Soc., 72 (1966), pp. 751-807.

[10] A. A. Himonas And G. MisioŁek, The Cauchy problem for an integrable shallow-water equation, Differential Integral Equations, 14 (2001), pp. 821-831.

[11] A. A. Himonas And G. MisioŁeK, High frequency smooth solutions and well-posedness of the Camassa-Holm equation, Int. Math. Res. Not., 51 (2005), pp. 3135-3151.

[12] G. MisioŁeK, Classical solutions of the periodic Camassa-Holm equation, Geom. Funct. Anal., 12 (2002), pp. 1080-1104.

[13] C. C. LIN, Note on a class of exact solutions in magnetohydrodynamics, Arch. Rational Mech. Anal., 1 (1958), pp. 391-395.

[14] Н. Окамото And J. Zhu, Some similarity solutions of the Navier-Stokes equations and related topics, Taiwanese J. Math., 4 (2000), pp. 65-103.

[15] J. T. StuART, Nonlinear Euler partial differential equations: Singularities in their solution, in Applied Mathematics, Fluid Mechanics, Astrophysics, World Scientific, Singapore, 1988, pp. 81-95.

[16] H. WEYL, On the differential equations of the simplest boundary-layer problems, Ann. of Math. (2), 43 (1942), pp. 381-407.

[17] W. Wolibner, Un theorème sur l'existence du mouvement plan d'un fluide parfait, homogene, incompressible, pendant un temps infiniment long, Math. Z., 37 (1933), pp. 698-726.

Copyright (c) by SIAM. Unauthorized reproduction of this article is prohibited. 